University of Nebraska - Lincoln

DigitalCommons@University of Nebraska - Lincoln

\title{
A Service-Differentiated Real-Time Communication Scheme for Wireless Sensor Networks
}

\author{
Yuyan Xue \\ University of Nebraska-Lincoln, yxue@cse.unl.edu \\ Byrav Ramamurthy \\ University of Nebraska-Lincoln, bramamurthy2@unl.edu \\ Mehmet C. Vuran \\ University of Nebraska-Lincoln, mcvuran@cse.unl.edu
}

Follow this and additional works at: https://digitalcommons.unl.edu/cseconfwork

Part of the Computer Sciences Commons

Xue, Yuyan; Ramamurthy, Byrav; and Vuran, Mehmet C., "A Service-Differentiated Real-Time Communication Scheme for Wireless Sensor Networks" (2008). CSE Conference and Workshop Papers. 107.

https://digitalcommons.unl.edu/cseconfwork/107

This Article is brought to you for free and open access by the Computer Science and Engineering, Department of at DigitalCommons@University of Nebraska - Lincoln. It has been accepted for inclusion in CSE Conference and Workshop Papers by an authorized administrator of DigitalCommons@University of Nebraska - Lincoln. 


\title{
A Service-Differentiated Real-Time Communication Scheme for Wireless Sensor Networks
}

\author{
Yuyan Xue, Byrav Ramamurthy and Mehmet C. Vuran \\ Department of Computer Science and Engineering \\ University of Nebraska-Lincoln \\ Lincoln, NE 68588-0115 USA \\ \{yxue, byrav, mcvuran\}@cse.unl.edu
}

\begin{abstract}
Supporting end-to-end real-time communication is important for wireless sensor networks (WSNs) to acomplish the collaborative sensing tasks with specific timing constraints. However, without considering the unique constraints for WSNs, many existing real-time communication protocols prove to be infeasible for low-cost WSNs. In this paper, we propose a novel real-time communication scheme (RCS) to provide servicedifferentiated soft real-time guarantees for end-to-end communication in WSNs. We use hop-based geographic grouping to enable location awareness for sensor nodes with extremely low control overhead. We use dynamic forwarding with load-balanced receiver contention to provide a light-weight, yet efficient, routing technique, which can be easily adapted for duty cycle design. We use polling contention period based real-time MAC support to improve the service-differentiation granularity with better bandwidth utilization. The performance evaluation shows that our scheme can achieve low end-to-end latency, high on-time delivery ratio, fine services-differentiation granularity with loadbalance for real-time traffic in unsynchronized low-cost WSNs.
\end{abstract}

\section{INTRODUCTION}

Supporting service-differentiated real-time communication is important for wireless sensor networks (WSNs) to achieve the collaborative sensing task with specific timing constraints. The timing constraints can arise for various reasons. For example, in an WSN event monitoring application, as shown in Fig. 1, the predefined events can be detected by the nearby sensor nodes. The collected event information needs to be sent to the sink within a certain period of time so that proper event response can be performed in a timely manner. According to the event urgency and importance, the data packets associated with different events can be assigned different end-to-end deadline requirements. Only the packets that are delivered to the sink before the deadline are deemed useful. Similar endto-end deadline requirements can be found in many delaysensitive WSN applications, such as multimedia surveillance, personal medical care, highway traffic coordination or remote robot control.

To support real-time communication in WSN, a prioritized medium access control (MAC) mechanism should be used for network traffic with different deadline requirements. The packets with tighter deadline requirements should get higher priority to access the wireless channel so that they can be delivered earlier to the destination. The IEEE 802.11 EDCA

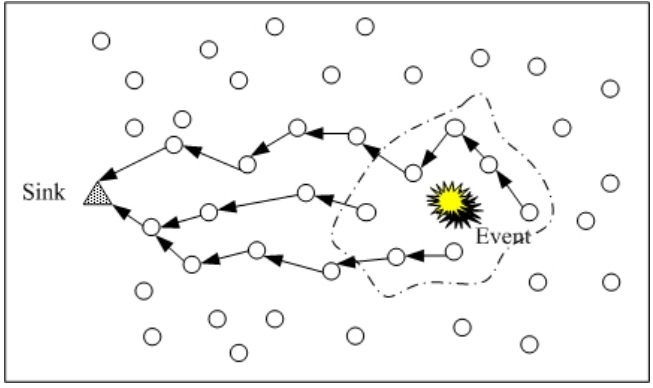

Fig. 1. Real-time traffic generated by dynamic event detection application.

(Enhanced Distributed Channel Access) standard [1] has been proposed as a solution for supporting service-differentiated delay-bounded traffic in WLAN (Wireless LAN). Many existing real-time communication frameworks for WSNs use the IEEE 802.11 EDCA as their MAC layer design [2][3][4]. We explain the limitations of these studies in Section II.

Routing is another major issue to be solved in designing a real-time communication scheme for WSNs. Compared with WLANs, where all the network nodes are assumed to communicate within the same interference area, WSNs require light-weight, yet efficient, routing schemes for end-to-end realtime communication. Both the end-to-end hop count and the channel quality along the route should be considered so that the real-time packets can be delivered in the least possible number of hops while achieving the least possible number of retransmissions across each hop.

Since the deadline requirements can be highly diverse for different events or applications, properly converting different deadline requirements into limited number of priority classes become a particular problem for WSNs. To provide Quality of Service (QoS) guarantees, IEEE 802.11e uses multiple queues for the handling of different traffic categories (TCs) [1]. The traffic is simply classified into high, medium and low priority. Each of these levels is associated with a FIFO (First In First Out) queue. The packets from different queues are assigned with different IFS (Inter Frame Space) and CW (Contention Widow) values so that they can get prioritized access to the wireless channel. In 802.11 EDCA, the medium and low priority TCs are further divided into sub-categories, where totally four TCs are supported [1]. Because WLANs are single-hop networks and usually have flow-based multimedia 
traffic, any packet that belongs to a flow will be assigned a single priority level according to its application types, such as data, voice, video and control. However, the timing constraints for WSN applications can be far more diverse. For example, under the event detection application, different event areas can generate traffic with different deadline requirements. In addition, even if the deadline requirements are the same, the end-to-end hop count can be significantly different for different events, thus introducing diverse per-hop deadline requirements. A simple priority assignment may not be able to meet the multi-hop real-time requirements in an end-to-end manner.

Finally, to prolong the life-time of a WSN while achieving the real-time communication requirements is vital to any feasible design for WSNs. Since the network lifetime can be deteriorated by either unbalanced energy utilization or an overwhelming amount of control overhead, we have to maintain the balance between end-to-end performance and the overall network resource consumption.

In the following sections, we propose a feasible servicedifferentiated Real-time Communication Scheme (RCS) for WSNs. We first discuss some existing real-time communication schemes for WSNs in Section II and point out their limitations. Accordingly, we give an overview and describe the detail operations of our real-time communication scheme, RCS, in Section III and IV. In Section V, we provide extensive simulation results for performance evaluation. The paper is concluded in Section VI.

\section{Motivation AND Related Work}

\section{A. MAC: TDMA or CSMA/CA}

Many solutions have been proposed for prioritized medium access control (MAC) in wireless networks [5][6][7]. These approaches can mainly be divided into two groups: reservation-based and contention-based [8]. Reservation-based schemes usually use TDMA based MAC operation. A strict requirement of these schemes is that the sensor network needs to be accurately synchronized, which introduces high control overhead for WSN applications. In addition, the signaling period required by TDMA schemes decreases the bandwidth utilization with increasing control overhead especially under light traffic load. Since we are seeking light-weight design for low-cost WSN applications, TDMA based MAC operation would not be a good choice.

Contention-based schemes usually use CSMA/CA (Carrier Sense Multiple Access/Collision Avoidance) with RTS/CTS based MAC operation, where the IFS and/or CW are modified according to different priority classes. Contention based schemes do not require network synchronization. However, since the length of IFS and CW affect the bandwidth utilization and the probability of correct priority schedule, proper IFSs and CWs for different priority classes and the number of priority classes in network should be determined to achieve better real-time capacity. Most recently proposed real-time communication schemes in WSNs [2] [3] [4] use IEEE 802.11e or 802.11 EDCA for differentiated MAC support. However, since most 802.11 based priority scheduling techniques try to prioritize the medium access by enlarging the IFSs and CWs for lower priority traffics, if many priority levels are supported by the network, the average IFS will become very large, which can lead to severely degraded throughput. The revised version of 802.11 EDCA cuts the supported number of priority levels from 8 to 4 mainly because of this reason. On the other hand, a smaller TC number will result in more traffic being assigned to the same priority, which increases the collision possibility among the transmissions. This will result in a larger $\mathrm{CW}$ value, which can deteriorate the overall communication throughput. A small TC number will also affect the service differentiation ability.

\section{B. Routing: Table based forwarding or dynamic forwarding technique}

Besides the prioritized MAC, different routing approaches have been employed in recently proposed real-time wireless communication schemes. RAP [4] uses a greedy geographic routing mechanism, where each packet can only be routed to the neighboring node with the shortest distance to the receiver. To this end, each sensor has to maintain a neighbor list with every neighbor's location information. The channel conditions are assumed to be perfect with minimal node mobility. Therefore, low control overhead can be achieved for neighbor list maintenance. One main drawback in the RAP design is that the greedy geographic forwarding does not consider the local network conditions in the next-hop selection. Therefore, RAP is hard to achieve load balance and congestion avoidance in packet forwarding. In addition, perfect channel condition is not a realistic assumption especially for low-power WSNs.

MMSPEED [2] and SPEED [3] also use neighbor lists for packet routing. Compared to RAP, these two protocols require the knowledge of both the location information and the average pairwise one-hop transmission delay for all neighboring nodes. Using the location and delay information, each node can evaluate the packet progress speed through each neighbor node towards the receiver and forwards a packet to a node whose progress speed is higher than the pre-specified lowerbound speed. SPEED and MMSpeed improve the routing design by considering the network conditions in choosing the route. However, both designs still require each sensor node to maintain a neighbor node information list for nexthop selection. Since whether the deadline requirements can be met for a packet is decided by the location and the delay information in the neighbor list, keeping the neighbor information up-to-date is vital for the design. Under the highly dynamic network conditions in WSNs, constantly exchanging the neighbor information will lead to huge control overhead, which in turn deteriorates the bandwidth utilization.

Another important problem with SPEED and MMSpeed is that both schemes are not suitable for duty cycle design, which is important for energy conservation in WSN. In an unsynchronized WSN, the sensor nodes with duty cycle design will randomly go to sleep mode to decrease the energy 
consumption. In this case, a table-based routing technique cannot properly identify the active next-hop candidate.

In contrast to table-based forwarding techniques, a dynamic forwarding technique has been proposed for multi-hop data communication in WSNs with minimal energy consumption, in recent studies [9] [10] [11]. The routing functionality is combined with the CSMA/CA based MAC design so that an adaptive receiver contention is performed at each hop. The sensor nodes with better forwarding distance, lower traffic load, higher channel quality or higher residual energy level will receive a higher priority to respond to the RTS packet and thus become the next hop. No routing tables or neighbor node information need to be maintained or periodically exchanged. Since the forwarding decision is made on-demand, it can easily adapt to a distributed duty-cycle design. The extra delay before CTS transmission for receiver contention is a tradeoff with increased control overhead. The existing dynamic forwarding technique motivates our light-weight communication protocol design by allowing for a cross-layer approach. Although it was originally proposed for reliable communication, if we can include the latency requirement into the receiver contention process and properly limit the extra delay period, this technique serve as a better routing scheme for real-time communication in WSNs.

\section{DESIGN OVERVIEW}

\section{A. Network Model}

We consider multi-hop wireless sensor networks with a single sink. The sensor nodes are homogenous unsynchronized devices without location awareness. All the sensor nodes are configured with uniform transmission power. The sink and sensor nodes communicate using a single channel. The above assumptions reflect the current hardware configurations of lowcost wireless sensor nodes [12].

We consider dynamic event detection for border control as our application. The predefined events are detected by the nearby sensor nodes and the event information is convergecasted to the sink [13]. According to the event urgency and importance, the data packets can be assigned different end-toend deadline requirements. Only the packets delivered to the sink before the deadline are deemed useful.

\section{B. Design Goal}

The main design goal of RCS is to support servicedifferentiated real-time communication for a WSN subject to the above network model. More specifically, our design satisfies the following objectives:

- Service-differentiated soft real-time guarantee: The proposed communication scheme should provide an accurate priority classification method and fine service differentiation granularity to dynamic event traffic with varying end-to-end deadline requirements. All packet arriving at the sink should be subject to the required end-to-end deadline. Proper admission control and early packet drop policy should be applied for achieving soft real-time guarantees.
- Minimum hardware support: The proposed communication scheme should work well on the low-cost sensor nodes with highly constrained memory and energy resources. No expensive localization/synchronization devices, such as GPS, or algorithms should be required.

- Adaptive to network dynamics: The proposed communication scheme should adapt well to the topology change due to node failure or duty cycle design. Therefore, a fully distributed decision process is required for packet forwarding. It should also consider the dynamic channel quality and traffic load for packet delivery, thus adapting well to channel fading and network congestion.

\section{Design Components}

In order to fulfill the design goals described in Section III.B for low-cost WSNs, we develope a real-time communication scheme consisting of four main components:

- Hop-based geographic sensor node grouping

- Per-hop deadline based prioritized queueing

- Polling contention period based real-time MAC

- Receiver contention based dynamic forwarding

The hop-based geographic grouping is designed for the postdeployment stage. The grouping results can help the sensor nodes obtain rough location awareness for better end-to-end latency estimation and accomplish routing decisions with low control overhead.

With the grouping information, the prioritized queueing policy is designed to classify the packet based on differentiated real-time requirements so that the packets with tighter deadline requirements can be scheduled to access the channel earlier.

The polling contention period based real-time MAC is then proposed to support prioritized channel access for the packets associated with different priority queues. Our real-time MAC is an improved design over 802.11 EDCA, which can help increase the service differentiation granularity with better overall bandwidth utilization. The receiver contention based dynamic forwarding is folded into the RTS/CTS exchanging process in real-time MAC for fully distributed on-demand routing. The real-time MAC and dynamic forwarding mechanism can guarantee that the packet with the highest priority level is delivered first to the best next-hop candidate so that the endto-end latency can be minimized based on a local decision.

\section{A Feasible Real-time Communication Scheme FOR WSNS}

\section{A. Hop-based geographic sensor node grouping}

Most existing real-time communication protocols for WSNs assume precise location awareness at each sensor node [3] [2], which requires GPS equipment or expensive localization schemes. In our scheme, we use a hop-based sensor node grouping to roughly strip the sensing field into layers. A similar approach is used for anchor beacon propagation for WSN localization [14]. The geographic grouping should be done at the post-deployment stage through a limited broadcasting. The grouping operations are given below: 
- The sink sets the same transmission power as the sensor nodes' and initializes a Grouping Message broadcast with group ID as 0 .

- The sensor nodes that receive the Grouping Message will assign their group ID as group ID in Grouping Message +1 . Any node that receives a group ID assignment rebroadcasts the grouping message with its own group ID once.

- The sensor nodes with lower group ID have less back-off time so that the sensor nodes with higher grouping ID cannot group the sensor nodes until all lower ID nodes broadcast their messages.

- The grouping broadcast stops when all the border nodes finish their broadcast.

After the hop-based grouping process, the sensor nodes can be grouped into strips with one-hop width. The density of the WSN will affect the grouping structure. With increasing node density, the result of grouping would approach perfect circular strips [15]. The group ID can be used to estimate the hop-distance from the node to the sink. With this grouping information, the packet forwarding can be guided towards the sink without precise location information. In a WSN, the node density is usually high enough to form near-circular groups. The grouping simulation results are shown in Section V. Compared with a precise localization scheme required in [2] and [3], the hop-based geographic grouping introduces much less control overhead and shorter initialization period, while providing accurate enough localization information for our dynamic forwarding scheme.

\section{B. Per-hop deadline based prioritized queueing policy}

In WSNs, an application-specific real-time requirement is usually presented as an end-to-end deadline, which indicates the maximum packet traversal time from the sender to the receiver [9]. However, in a multi-hop network, the end-to-end deadline is not the only criterion to determine the urgency of packet delivery. The end-to-end hop count also affects the packet delivery schedule. For example, if there are two packets with the same end-to-end deadline requirements competing for the channel, the one with higher end-to-end hop count should be scheduled first. If we assume that the geographic grouping would provide each sensor with accurate enough hop-count to the sink, the end-to-end deadline requirement can be broken down into a per-hop deadline requirement, where

$$
\text { Deadline }_{\text {per-hop }}=\frac{\text { Deadline }_{\text {End-to-End }}}{\text { Sender's GroupID }^{\text {Sende }}}
$$

Deadline $_{\text {per-hop }}$ reflects the required per-hop traversal speed to achieve the end-to-end real-time guarantee in contention-

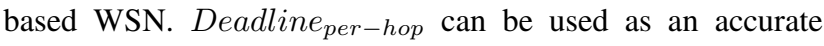
enough indicator for packet delivery priority classification [16].

We use FIFO priority queue for packet scheduling at a node. Since the prioritized MAC can only provide differentiated service for a limited number of priority classes, the perhop deadline requirements are further mapped into $N$ priority levels. The priority level for a packet $P$ can be derived by

$$
P= \begin{cases}\left\lfloor\frac{\text { Deadline }_{\text {per-hop }}^{\text {Req }}}{\text { Deadline }} \text { per-hop }_{\text {Min }}^{\text {Din }}\right\rfloor, & P<N, \\ N, & \text { else, }\end{cases}
$$

where the Deadline per-hop $_{\text {in }}$ is calculated based on our realtime MAC operation, which will be introduced in Section IV.D. Each sensor node is allocated $N$ priority queues so that every incoming packet can be classified and placed into the proper priority queue for transmission. Since Early Deadline First (EDF) has been proven as the most efficient scheduling policy for channel access in wireless networks [7], the packet with a smaller $N$ level will be scheduled first for transmission.

Sender's GroupID*Deadline per-hop $_{\text {in }}$ represents the best possible end-to-end delay for a packet, all packets, that can be delivered to the sink before the deadline should have Deadline per-hop $_{\text {Req }} \geq$ Deadline per-hop $_{\text {Min }}$. Accordingly, we design a simple admission control and early deadline-miss drop policy at the sending and relaying nodes. At the sending node, if the packet's Deadline per-hop $_{\text {is mapped into a priority level }}$ less then 1, the packet will not be admitted into the network. At each relaying node, the cumulative packet transmission time will be recorded as $t_{A}$ and the remaining deadline for a packet will be calculated, where

$$
\text { Deadline }_{\text {remain }}=\text { Deadline }_{\text {End-to-End }}-t_{A}
$$

The updated per-hop deadline will be calculated at each relaying node based on

$$
\text { Deadline }_{\text {per-hop }}=\frac{\text { Deadline }_{\text {remain }}}{\text { Relayer's GroupID }_{\text {Leup }}}
$$

If the packet's updated Deadline per-hop is mapped into a priority level less then 1 , the packet will be dropped because it is unlikely to be delivered to the sink on time.

In contrast to the packet drop policy adopted by MMSpeed [2] or SPEED [3], which depend on the periodically updated per-hop delay information stored in neighbor list, this early drop policy can better adapt to the highly dynamic channel and load conditions in WSNs. It can avoid false packet drops due to outdated per-hop pairwise delay information.

\section{Polling contention period-based real-time MAC}

In order to better support the diverse end-to-end deadline requirements in WSN applications, we propose a polling contention period based real-time MAC to support prioritized channel access.

The 802.11 EDCA is used by most existing real-time communication schemes for prioritized MAC support in WSNs. It uses extended AIFS and contention window (CW) for prioritized medium access contention. For a packet with priority level $i$, the AIFS value will be derived as follows [1]:

$$
\begin{array}{r}
A I F S_{i}=S I F S+i * S L O T \_T I M E \\
C W_{i}=\left(C W_{1}+1\right) * i-1
\end{array}
$$

where SIFS is Short Inter Frames for control packet transmission contention. According to the 802.11 EDCA design, 


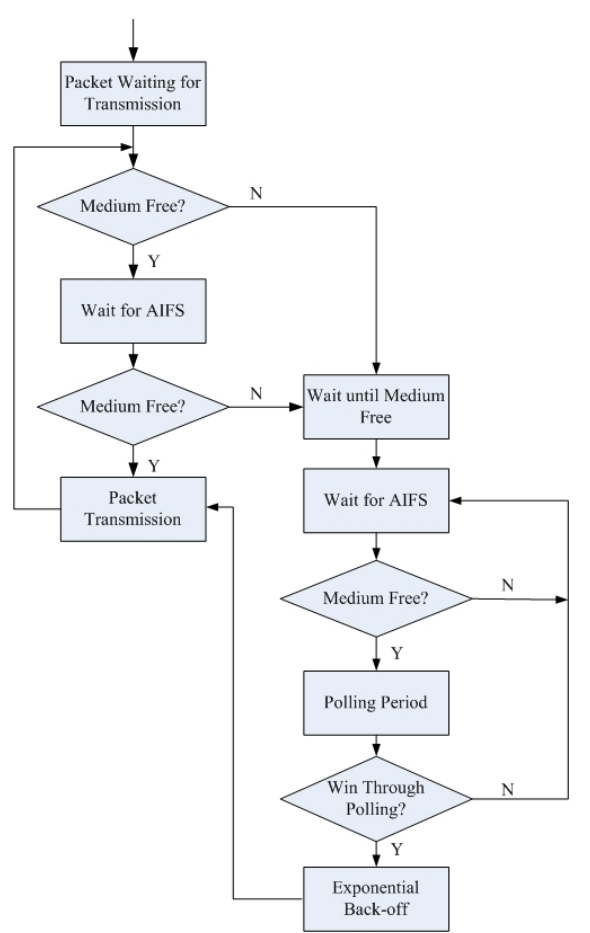

Fig. 2. Polling period-based transmission contention in Real-time MAC

the higher the number of priority levels supported in the network, the longer are the average AIFS and back-off window values that are used in the MAC operation, and the less the average throughput that can be achieved in terms of overall transmission performance.

TABLE I

Polling-SLOT DESIGN FOR MAXIMUM PRIORITy LEVEL $=7$

\begin{tabular}{|c|c|c|c|}
\hline Priority Level & Slot 1 & Slot 2 & Slot 3 \\
\hline 7 & active & active & active \\
\hline 6 & active & active & inactive \\
\hline 5 & active & inactive & active \\
\hline 4 & active & inactive & inactive \\
\hline 3 & inactive & active & active \\
\hline 2 & inactive & active & inactive \\
\hline 1 & inactive & inactive & active \\
\hline
\end{tabular}

In RCS, we use a fixed number of polling slots instead of extended IFS for prioritized packet transmission contention, as shown in Fig. 2. This idea is motivated by the bus access control mechanism in a computer system, where $\lfloor\sqrt{N+1}\rfloor$ polling slots are required for contention entities with $N$ priority levels. For example, if 7 priority levels are supported by our design, 3 polling slots are required for medium access contention among all possible competitors within the interference area. According to Table I, the nodes with a current packet at priority level $i$ will transmit a burst signal in their active polling slots and keep silent in their inactive polling slots. Any node that senses a burst in its inactive polling slots will be suppressed in the following transmission period. In this manner, only the node with the highest priority level among all competitors can enter the back-off period for RTS transmission. As a result, the number of competing nodes will dramatically decrease after the polling competition period and this results in less collision possibility; thus the $\mathrm{CW}$ can be set to a smaller size compared with that in 802.11 EDCA.

If we assume that all priority levels have the same amount of traffic load, with polling competition period design, our real-time MAC can result in better overall throughput when the number of priority levels satisfies

$$
\sqrt{N+1}<N \Rightarrow N>2
$$

without considering the throughput gain by the possible smaller CW.

\section{Receiver contention-based dynamic forwarding}

According to our feasibility analysis in Section II, we use receiver contention-based dynamic forwarding for convergecast packet routing. This idea comes from the early work proposed in [9] and [10], while we used different metrics for next-hop selection to best fit into our design for real-time constraints. The dynamic forwarding process is combined into the RTS/CTS exchanging period of real-time MAC design.

According to the real-time MAC design, if a sender wins during a polling contention period and gains the medium access after the exponential back-off period, it will initiate an RTS transmission containing its own groupID, $G_{T x}$. All the nodes within the transmission range will overhear this RTS message and enter the receiver contention period.

In the receiver contention period, only the sensor nodes with the same or lower groupID to $G_{T x}$, become the qualified next-hop candidates so that the packet can only be forwarded towards the sink to gain non-negative packet traverse speed. The unqualified nodes enter the NAV (Network Allocation Vector) period. Every qualified next-hop candidate is required to evaluate its capability of minimizing the per-hop delivery latency for this transmission. The capability is classified into $M$ priority levels for receiver contention. The contention priority levels $P_{C T S}$ is derived based on a potential receiver's average packet transmission time $t_{\text {trans }}^{\text {Avg }}$, queuing length $L_{Q}$ and groupId $G_{R x}$, and shown as follows:

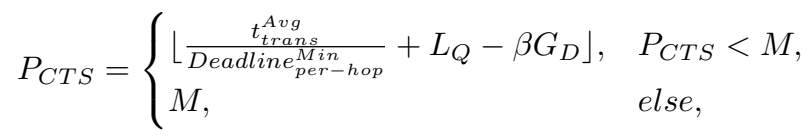

where $G_{D}=1-G_{R x}+G_{T x}$.

$t_{\text {trans }}^{\text {Avg }}$ is calculated using a weighted moving average of the instantaneous packet transmission time $t_{\text {trans }}$ as

$$
t_{\text {trans }}^{\text {Avg }}=\alpha t_{\text {trans }}+(1-\alpha) t_{\text {trans }}^{\text {Avg }}
$$

For each packet transmission, the instantaneous packet transmission time is measured from the time an RTS is transmitted to the time the corresponding ACK is received. If the packet is dropped due to exceeding the maximum retransmission time, $T_{\text {Re_Trans }}$,

$$
t_{\text {trans }}=\text { Deadline }_{\text {per-hop }}^{\text {Min }} * T_{\text {Re_Trans }}
$$


Fig. 3. Prioritized packet transmission contention in Real-time MAC.

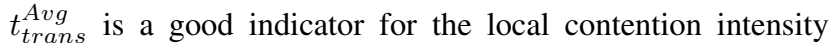
and channel quality (error rate) of a potential receiver. A

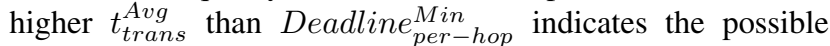
retransmission time per packet transmission.

The queuing length $L_{Q}$ reflects the traffic load at a particular sensor node. A larger queuing length indicates longer queuing delay before a packet can be scheduled for transmission.

The groupId difference between the sender and receiver $G_{D}$ reflects the end-to-end hop progress for a forwarding decision. Since all the sensor nodes are pre-grouped using hop-based geographic grouping, for any possible forwarding decision, we have $0 \leq G_{D} \leq 1$. $\beta$ is a scalar for tuning the receiver contention preference between lower groupId and lower per-hop transmission delay, where $1 \leq \beta \leq 6$. A larger $\beta$ provides a higher possibility for a more aggressive forwarding towards the sink compared with a routing decision within the same group for achieving load-balance.

The above receiver contention-based priority assignment guarantees that

- The sensor node with a lower group ID will receive a higher priority for transmitting its CTS packet.

- For the sensor node with the same group ID, the sensor node with a better channel quality and less traffic load will get a higher priority for transmitting its CTS packet.

Upon receiving the RTS packet, each possible next-hop candidate will first wait for an SIFS period and compete in $\sqrt{M+1}$ polling period according to its priority level. The winner enters an extra back-off period for possible collision among the candidates with the same contention priority or that cannot hear the polling slots from each other. Since the collision probability is very low after the polling contention period, the back-off period is usually set to a much smaller number compared with $C W_{R T S}$. After the extra back-off period, the winning receiver sends back the CTS packet with its sensor ID to notify the sender.

A complete prioritized packet contention and receiver contention period for real-time MAC is shown in Fig. 3. The minimum per-hop latency for the packet transmission with any priority level assignment can be derived accordingly as

$$
\begin{array}{r}
\text { Deadline }_{\text {per-hop }}^{\text {min }}= \\
\text { AIFS }+t_{\text {Polling }}^{\text {RTS }}+\frac{1}{2} C W_{\text {RTS,min }}+t_{\text {RTS }} \\
+S I F S+t_{\text {Data }} \\
+ \text { SIFS }+t_{\text {Polling }}^{\text {CTS }}+\frac{1}{2} C W_{C T S, \text { min }}+t_{C T S} \\
+S I F S+t_{A C K}
\end{array}
$$

where, AIFS and SIFS are arbitrary and short IFSs; $t_{P o l l i n g}^{R T S}$ and $t_{\text {Polling }}^{C T S}$ are fixed time of polling period for RTS and
CTS packets; $C W_{R T S, \text { min }}$ and $C W_{C T S, \text { min }}$ are minimum contention window value; $t_{R T S}, t_{C T S}, t_{D a t a}$ and $t_{A C K}$ are RTS, CTS, Data and ACK packet transmission time separately.

\section{Performance Evaluation}

The performance of the developed real-time communication scheme, RCS, is analyzed in GlomoSim [17], a simulation environment for wireless network, using the simulation parameters shown in Table II. The simulation parameters are carefully chosen to reflect typical wireless sensor node capabilities. We conduct extensive simulation scenarios for RCS and compare its performance with existing real-time communication schemes, RAP [4] and MMSpeed [2]. Since the original MMSpeed design try to target both end-to-end delay and end-to-end reliability requirements, it performs multicast on each intermediate nodes for reliability enforcement. We did not implement this feature in our simulations to make MMSpeed comparable to our design in targeting only realtime performance and more energy efficient. We rename the simplified version of MMSpeed as MMSpeed*. The MAC operation parameters for both 802.11 EDCA (used by RAP and MMSpeed), and real-time MAC (used by RCS), are listed in Table III. For each simulation scenario, we conduct the simulation 10 times with different random seeds. We use the average value collected from all 10 simulations for performance evaluation.

TABLE II

\begin{tabular}{|c|c|c|}
\hline & 802.11 EDCA & Real-time MAC \\
\hline Retransmission Limit & 7 & 7 \\
\hline Priority Class & 4 & 7 \\
\hline SIFS & $10 \mu \mathrm{s}$ & $10 \mu \mathrm{s}$ \\
\hline Time Slot & $20 \mu \mathrm{s}$ & $20 \mu \mathrm{s}$ \\
\hline AIFS[1] & $30 \mu \mathrm{s}$ & $80 \mu \mathrm{s}$ \\
\hline$C W_{R T S}^{M i n}[1]$ & 15 Slots & 10 Slots \\
\hline$C W_{R T S}^{\operatorname{Max}}[1]$ & 255 Slots & 200 Slots \\
\hline$C W_{C T S}$ & N/A & 4 Slots \\
\hline
\end{tabular}

Simulation ENVIRONMENT SETTINGS

\begin{tabular}{|c|c|}
\hline Sensing field dimensions & $(500 \times 500) \mathrm{m}$ \\
\hline Sink location & $(10,15)$ \\
\hline Number of sensor nodes & 100 \\
\hline Node placement & Uniform \\
\hline Sensor node radio range & $110 \mathrm{~m}$ \\
\hline Packet length & 128 bytes \\
\hline Radio bandwidth & 250 kbps \\
\hline Channel model & Random two-ray \\
\hline
\end{tabular}

TABLE III

802.11EDCA AND REAL-TIME MAC PARAMETERS

In the first simulation scenario, we examine whether the hop-based geographic grouping can properly provide the rough 


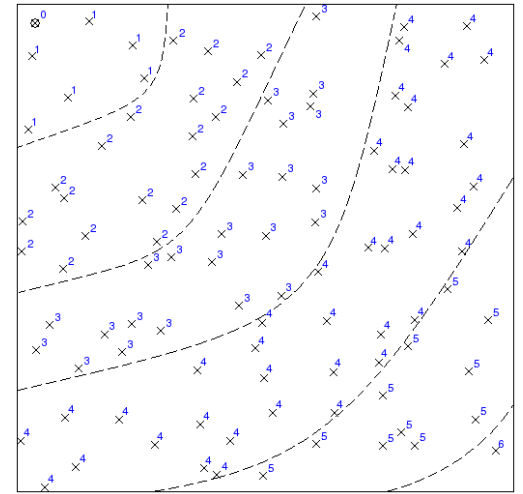

Fig. 4. Group formation results based on the simulation network topology.

location information for each sensor node in RCS. Fig. 5 shows the network topology generated for the simulation and the results of hop-based geographic grouping based on the topology. From the grouping result, we can conclude that with enough network density $\left(15 /\left(\pi *\right.\right.$ RadioRange $\left.^{2}\right)$ in simulation), simple hop-based geographic grouping can properly divide the sensing field into near circular strips. The groupId assigned to the sensor node can correctly indicate the rough hop-distance from the sensor node to the sink.

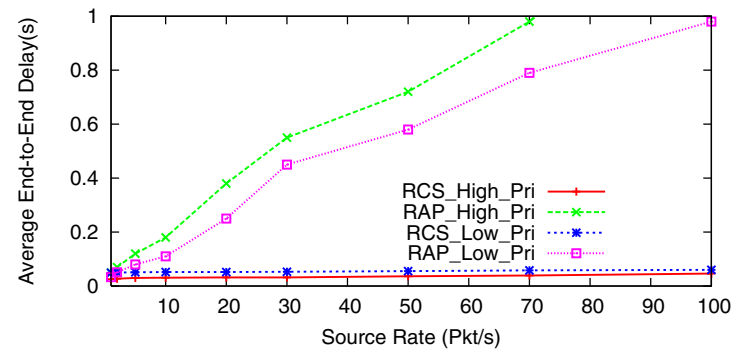

(a) RCS VS. RAP

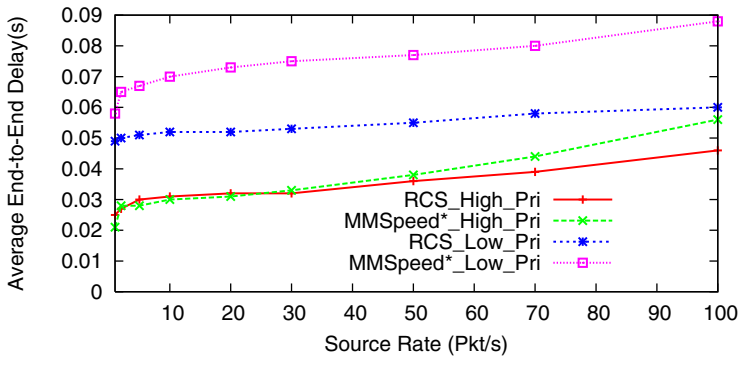

(b) RCS VS. MMSpeed*

Fig. 5. End-to-end delay for two event data flows with different priority levels generated by two nodes with GroupId $=5$.

In the second simulation scenario, we compare the average end-to-end delay achieved by RCS, RAP and MMSpeed*. In order to test the performance of dynamic forwarding using groupID, we chose two nodes located in the left-bottom corner as the event data sources for maximizing the possible endto-end hop count. Two CBR (constant bit rate) event data flows CBR1 and CBR2 are generated from two nodes both with GroupId $=5$. CBR1 is assigned a tighter end-to-end deadline requirement of $40 \mathrm{~ms}$. CBR2 is assigned with a looser end-to-end deadline requirement of $80 \mathrm{~ms}$. According to RCS design, Priority ${ }_{C B R 1}=2$ and Priority PBR $_{B}=4$. Since both RAP and MMSpeed* design do not support dynamic deadline/priority level conversion for an application, we manually set the same priority level derived by RCS to RAP and MMSpeed*.

Fig. 5 gives the average end-to-end delays comparison among RAP, RCS and MMSpeed*. From the simulation results, we note that RCS provides much better end-to-end delay compared to RAP for both event data flows, because the pure geographic forwarding used in RAP cannot adapt to channel quality dynamics. In our simulation environment setting, a random two-ray channel model is used to reflect the channel dynamics in real WSN deployments. Therefore, the channel quality along the route, instead of the priority level, dominates the end-to-end delay for both flows in RAP. For this reason, RAP fails to provide service differentiation for these two data flows and results in a higher end-to-end delay for CBR1. In contrast to MMSpeed*, from Fig. 5(b), RCS provides similar end-to-end delay for high priority data flows and outperforms MMSpeed* for low priority data flows. Since both MMSpeed* and RCS consider the dynamic network conditions in packet forwarding, the simulation results show that both protocols can adapt well to channel fading. In addition, compared with the dynamic forwarding based on precise location information in MMSpeed*, the dynamic forwarding using groupID can achieve similar performance in RCS. RCS provides better endto-end delays for low priority traffic because the 802.11 EDCA extends the AIFS and CW value for CBR2, which leads to a deteriorated end-to-end throughput.

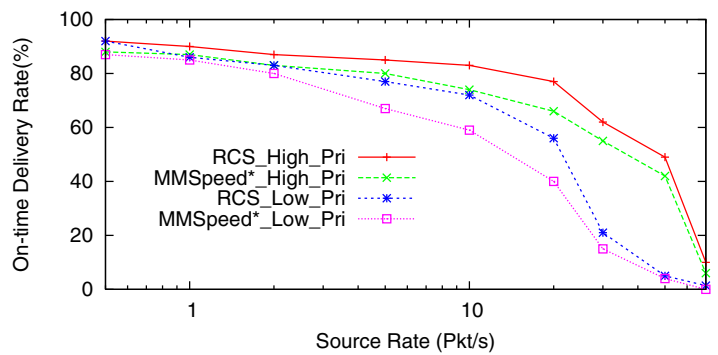

Fig. 6. On-time delivery rates for two event data flows with different priority levels generated by two nodes with GroupId $=5$.

Fig. 6 shows the on-time delivery ratio comparison for CBR1 and CBR2 using RCS and MMSpeed*. The application scenario remains the same as in the last simulation. The simulation results show that RCS overcomes MMSpeed* in on-time delivery ratio for both priority levels. The reason behind the results lies in that the rigid packet-drop policy adopted by MMSpeed* cannot work well based on the periodically updated per-hop delay information in the neighbor list. Therefore, certain amount of deliverable packets are dropped on the intermediate node due to the early packet drop policy. The simulation results further prove that it is hard to maintain 
the balance between control message exchanging overhead and freshness of the neighbor list for the table-based forwarding technique.

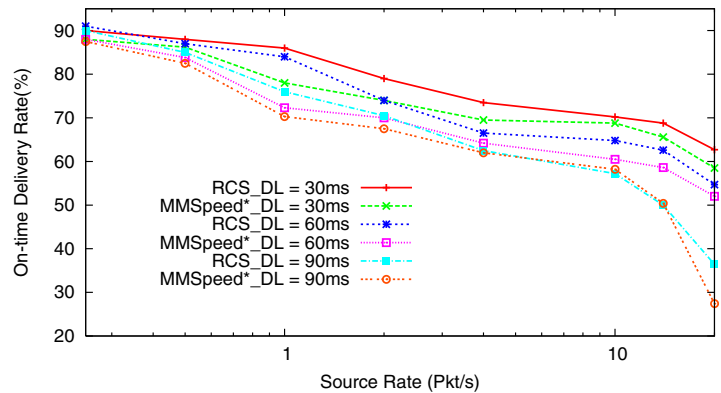

(a) On-time delivery ratio

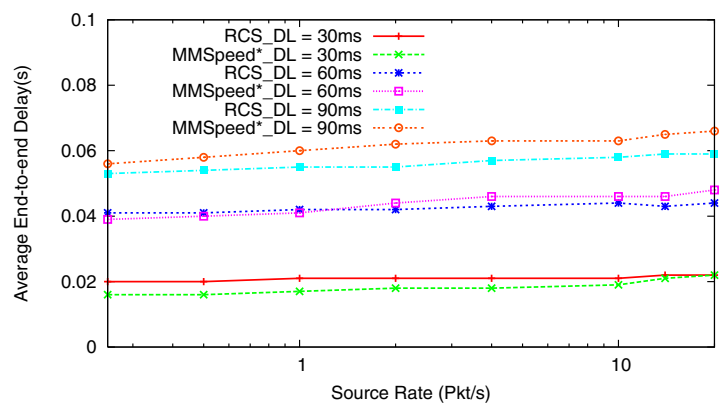

(b) Average end-to-end delay

Fig. 7. Service differentiation capability provided by RCS and MMSpeed* in terms of on-time delivery ratio and average end-toend delay.

In the third simulation scenario, we show the service differentiation capability provided by RCS and MMSpeed* in terms of on-time delivery ratio and average end-to-end delay. Three CBR event data flows, CBR1 (end-to-end deadline = $30 \mathrm{~ms}$ ), CBR2 (end-to-end deadline $=60 \mathrm{~ms}$ ) and CBR3 (endto-end deadline $=90 \mathrm{~ms}$ ) are generated from three nodes with GroupId $=5$. For RCS, the three deadline requirements are automatically mapped into priority levels 1,3 and 5 according to per-hop deadline-based queuing policy. In Fig. 7, it is shown that RCS can provide fine service differentiation for the traffic with different deadline requirements while keeping the fairness throughput allocation for prioritized traffic in terms of on-time delivery ratio. For MMSpeed*, we have to manually map the deadline requirements into priority levels 1,3 and 4 , since there are only 4 priority levels supported by 802.11 EDCA. From the simulation results, we conclude that although MMSpeed* can also provide service differentiation for CBR1, CBR2 and CBR3, the differentiation granularity is adversely affected in terms of deadline/priority mapping. If more than 4 deadline requirements arise in the network, MMSpeed* cannot provide service differentiation for the traffic while maintaining an overall throughput comparable to that of RCS.

\section{CONClusion AND Future WORK}

In this paper, we proposed a novel design to provide service differentiated soft real-time guarantees for end-to- end communication in low-cost WSNs. The proposed design requires minimum hardware support at the sensor nodes and adapts well to network dynamics. According to the design and performance analysis, our real-time communication scheme RCS is shown to achieve low end-to-end latency, better ontime delivery ratio, fine service-differentiated granularity with load-balance for real-time traffic in unsynchronized WSNs. Our future work includes extending the current scheme with duty cycle design, implementing the design in a sensor network testbed and applying the data generated by real event detection applications for further performance evaluation and improvement.

\section{REFERENCES}

[1] IEEE802.11WG, "Draft supplement to IEEE standard802.11-1999: Medium access control (MAC) enhancements for quality of service (QoS)," 2003.

[2] E. Felemban, C. Lee, and E. Ekici, "MMSPEED: Multipath multi-speed protocol for qos guarantee of reliability and timeliness in wireless sensor networks," IEEE Transactions on Mobile Computing, vol. 5, no. 6, pp. 738-754, June 2006.

[3] T. He, J. Stankovic, C. Lu, and T. Abdelzaher, "A spatiotemporal communication protocol for wireless sensor networks," IEEE Transactions on Parallel and Distributed Systems, vol. 16, pp. 995-1006, May 2005.

[4] C. Lu, B. Blum, T. Abdelzaher, J. Stankovic, and T. He, "RAP: A real-time communication architecture for large-scale wireless sensor networks," in Proc. of IEEE Real-Time and Embedded Technology and Applications Symp. (RTAS 2002), September 2002.

[5] I. Rhee, A. Warrier, J. Min, and L. Xu, "DRAND: distributed randomized TDMA scheduling for wireless ad-hoc networks," in Proc. of the 7th ACM international symposium on Mobile ad hoc networking and computing (MobiHoc 2006), New York, NY, USA, May 2006, pp. 190 201.

[6] B. D. Bui, R. Pellizzoni, M. Caccamo, C. F. Cheah, and A. Tzakis, "Soft real-time chains for multi-hop wireless adhoc networks," in Proc. of IEEE Real-Time and Embedded Technology and Applications Symp. (RTAS 2007), April 2007.

[7] Y. Tian, A. Dogan, and F. Özgüner, "An urgency-based prioritized mac layer protocol for real-time traffic in ad-hoc wireless networks," in Proc. of the 17th International Symposium on Parallel and Distributed Processing, Washington, USA, April 2003.

[8] I. Aad and C. Castelluccia, "Differentiation mechanisms for IEEE 802.11," in Proc. of IEEE INFOCOM 2001, vol. 1, April 2001, pp. 209-218.

[9] I. F. Akyildiz, M. C. Vuran, and O. B. Akan, "A cross layer protocol for wireless sensor networks," in Proc. of Conference on Information Sciences and Systems (CISS '06), Princeton, NJ, March 2006.

[10] P. Casari, M. Nati, C. Petrioli, and M. Zorzi, "ALBA: An adaptive load-balanced algorithm for geographic forwarding in wireless sensor networks," in Proc. of IEEE MILCOM 2006, September 2006.

[11] P. Casari, M. Nati, C. Petrioli, et al., "Efficient non-planar routing around dead ends in sparse topologies using random forwarding," in Proc. of IEEE ICC '07, June 2007.

[12] "MicaZ wireless module." [Online]. Available: http://www.xbow.com/ Products/Product_pdf_files/Wireless_pdf/MICAz_Datasheet.pdf

[13] H. Zhang, A. Arora, Y. Choi, et al., "Reliable bursty convergecast in wireless sensor networks," Computer Communication, vol. 30, no. 13, pp. 2560-2576, 2007.

[14] D. Niculescu and B. Nath, "DV based positioning in ad hoc networks," Journal of Telecommunication Systems, vol. 22, no. 1-4, January 2003.

[15] S. Vural and E. Ekici, "Hop-distance based addressing and routing for dense sensor networks without location information," Ad Hoc Netw., vol. 5, no. 4, pp. 486-503, 2007.

[16] T. F. Abdelzaher, S. Prabh, and R. Kiran, "On real-time capacity limits of multihop wireless sensor networks," in Proc. of IEEE RTSS 2004, December 2004.

[17] X. Zeng, R. Bagrodia, and M. Gerla, "GloMoSim: A library for parallel simulation of large-scale wireless networks," in Workshop on Parallel and Distributed Simulation, May 1998, pp. 154-161. 